\title{
ANALYTICAL AND NUMERICAL RESULTS FOR FLOW AND SHOCK FORMATION IN TWO-LAYER GRAVITY CURRENTS
}

\author{
P. J. MONTGOMERY ${ }^{1}$ and T. B. MOODIE
}

(Received 10 December 1996; revised 10 December 1996)

\begin{abstract}
Many gravity driven flows can be modelled as homogeneous layers of inviscid fluid with a hydrostatic pressure distribution. There are examples throughout oceanography, meteorology, and many engineering applications, yet there are areas which require further investigation. Analytical and numerical results for two-layer shallow-water formulations of time dependent gravity currents travelling in one spatial dimension are presented. Model equations for three physical limits are developed from the hydraulic equations, and numerical solutions are produced using a relaxation scheme for conservation laws developed recently by S. Jin and X. Zin [6]. Hyperbolicity of the model equations is examined in conjunction with the stability Froude number, and shock formation at the interface of the two layers is investigated using the theory of weakly nonlinear hyperbolic waves.
\end{abstract}

\section{Introduction}

A gravity current consists of one fluid flowing within another when this flow is driven by relatively small density differences between the fluids. Gravity currents are primarily horizontal occurring as either top or bottom boundary currents or as intrusions at some intermediate level, although there are important examples in oceanography, meteorology and elsewhere in which these currents have distinct vertical components. A thorough description of the numerous and various manifestations of gravity currents has been completed by Simpson [14].

The vast majority of the theoretical work on gravity currents from the time of von Kármán [9] up to Benjamin [1] and right on through the 1980s, treated the gravity current as steady, existing in either an inertia-buoyancy balance or, at later stages in the flow when the bottom gravity current had thinned out, in a viscous-buoyancy balance. A notable exception was the work by Hoult [7] in which governing equations

\footnotetext{
'Applied Mathematics Institute, Department of Mathematical Sciences, University of Alberta, Edmonton, Alberta, Canada

(c) Australian Mathematical Society, 1998, Serial-fee code 0334-2700/98
} 
were established and subsequently solved rather than seeking a balance of forces. His solution was obtained in terms of a similarity variable so that the derived relationships are valid only once sufficient time has elapsed after the release of a fixed volume of fluid, for the initial geometry of the volume to be irrelevant. One of the model systems to be presented here has been subjected to a similarity analysis in an earlier paper [3] and these similarity solutions will be used as a comparison with computed solutions.

The plan of this paper is as follows. In Section 2 we formulate the system of equations prescribing both mass and momentum balance in each layer together with the coupling between layers that results from buoyancy forces acting on a sloping density interface. We then specialize these equations to describe three distinguished limits of interest. These are the weak stratification limit [3] and the limits of a thin lower or upper layer. Section 3 describes the application of a numerical scheme for conservation laws [8] to both the general equations and the three physical limits. This numerical scheme is second order, non-oscillatory, converges to the correct weak solution for conservation laws and is straightforward to implement in this case. In Section 4 the model systems are examined in light of recent results of Hoff [6] concerning invariant regions associated with systems of conservation laws. We find that the region of hyperbolicity for our weak stratification model is invariant, and that it coincides with the region of subcritical flow depending on the stability Froude number as defined by Lawrence [10]. Section 5 is devoted to a discussion of shock formation at the density interface and Section 6 contains a few concluding remarks.

\section{The Model Equations}

The physical configuration of our two-layer fluid system is depicted in Figure 1. The fluids are assumed inviscid and of constant density, and we neglect the effects of both the Coriolis force and surface tension at the interface. In Figure 1, $\eta(x, t)$ represents the displacement of the upper surface from its undisturbed state, $\mathbf{u}=(\boldsymbol{u}, w)$ is the fluid velocity for plane flow in Cartesian coordinates with position vector $\mathbf{x}=(x, z)$, $H$ is the mean total depth, $h(x, t)$ is the thickness of the lower fluid, and $\rho_{1}, \rho_{2}$ (with $\rho_{1}<\rho_{2}$ ) represent the stably stratified densities of the upper and lower fluid layers respectively.

Employing the standard scaling arguments of shallow water theory based upon the smallness of the aspect ratio [13] we find the horizontal velocities, $u_{1}$ in the upper layer and $u_{2}$ in the lower layer, to be independent of height so that $u_{1,2}=u_{1,2}(x, t)$, the pressure field to be hydrostatic, and the dimensional governing equations to be

$$
\frac{\partial u_{1}}{\partial t}+u_{1} \frac{\partial u_{1}}{\partial x}+g \frac{\partial \eta}{\partial x}=0
$$




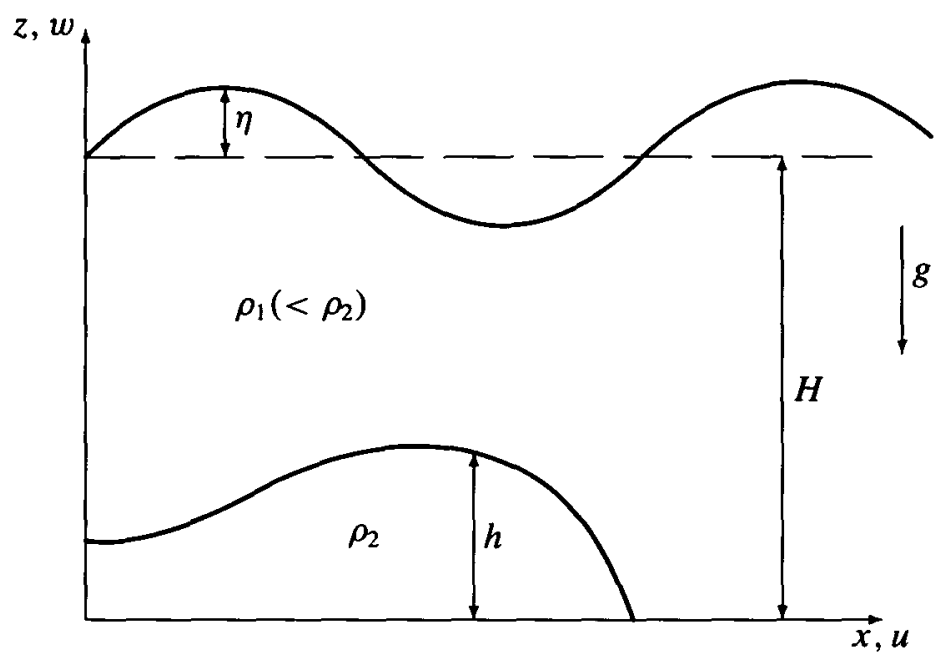

FIGURE 1. The two-layer fluid model.

$$
\begin{gathered}
\frac{\partial}{\partial t}(\eta-h)+\frac{\partial}{\partial x}\left[(H+\eta-h) u_{1}\right]=0 \\
\frac{\partial u_{2}}{\partial t}+u_{2} \frac{\partial u_{2}}{\partial x}+\left(g-g^{\prime}\right) \frac{\partial \eta}{\partial x}+g^{\prime} \frac{\partial h}{\partial x}=0 \\
\frac{\partial h}{\partial t}+\frac{\partial}{\partial x}\left(h u_{2}\right)=0 .
\end{gathered}
$$

In deriving these equations we have employed the usual kinematic conditions at the free surface and the interface, with closure being achieved by invoking continuity of the hydrostatic pressure field across the interface. The reduced gravity appearing in (2.3) is defined by $g^{\prime}=\left(\rho_{2}-\rho_{1}\right) g / \rho_{2}$.

Equations (2.1)-(2.4) are often referred to as the hydraulic equations and are equivalent to those given in [10]. In addition, these equations may be regarded as a generalization of those derived in [2] for the two-layer fluid model: our equations do not make any restrictions on the overall height of the two fluid layers, and as such the surface deflection term $\eta$ is retained.

2.1. Weak-Stratification Model We render equations (2.1) to (2.4) nondimensional by using a scaling that focuses on the nonlinear internal gravity wave processes. To this end we introduce nondimensional quantities signified by a tilde according to

$$
\eta=\frac{g^{\prime}}{g} H \tilde{\eta}, \quad\left(u_{1}, u_{2}\right)=\sqrt{g^{\prime} H}\left(\tilde{u}_{1}, \tilde{u}_{2}\right), \quad h=H \tilde{h}, \quad L / T=\sqrt{g^{\prime} H},
$$


where $L$ is a horizontal length scale and $T$ a time scale which are chosen so that their ratio is $\sqrt{g^{\prime} H}$. Under this advective scaling the nondimensional equations arising from substituting (2.5) into (2.1) to (2.4), are written in conservation form as

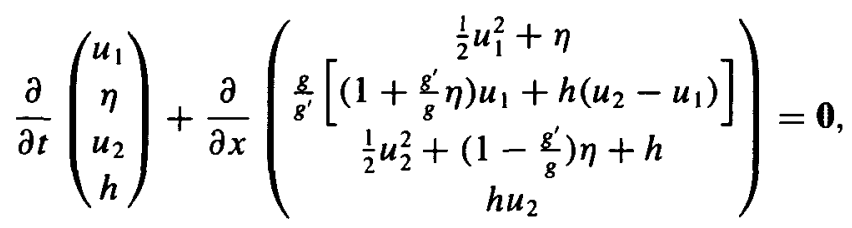

where the tildes have been dropped for notational convenience. The nondimensional equations (2.6) are now in a form similar to those for the three-layer model derived in [4], once the appropriate reduction to two layers has been made.

To achieve our weak-stratification model we neglect terms $O\left(g^{\prime} / g\right)$ in (2.6) on the assumption that density differences are small (that is, the system is Boussinesq) to write the equations

$$
\begin{gathered}
\frac{\partial u_{1}}{\partial t}+u_{1} \frac{\partial u_{1}}{\partial x}+\frac{\partial \eta}{\partial x}=0 \\
\frac{\partial}{\partial x}\left[u_{1}+h\left(u_{2}-u_{1}\right)\right]=0 \\
\frac{\partial u_{2}}{\partial t}+u_{2} \frac{\partial u_{2}}{\partial x}+\frac{\partial \eta}{\partial x}+\frac{\partial h}{\partial x}=0
\end{gathered}
$$

and

$$
\frac{\partial h}{\partial t}+\frac{\partial}{\partial x}\left(h u_{2}\right)=0
$$

If we invoke the zero mass flux condition at $x=0$, we may integrate (2.8) to produce the condition of no net mass flux,

$$
(1-h) u_{1}+h u_{2}=0 .
$$

The condition of no net flux of linear momentum,

$$
(1-h) u_{1}^{2}+h u_{2}^{2}+\eta+\frac{1}{2} h^{2}=0,
$$

can also be produced via the following manipulation. The sum of (2.7).multiplied by $(1-h),(2.9)$ multiplied by $h$ and (2.10) multiplied by $u_{2}-u_{1}$ gives

$$
\begin{gathered}
\frac{\partial}{\partial t}\left[(1-h) u_{1}+h u_{2}\right]+(1-h) u_{1} \frac{\partial u_{1}}{\partial x}-u_{1} \frac{\partial}{\partial x}\left(h u_{2}\right) \\
+\frac{\partial}{\partial x}\left(h u_{2}^{2}\right)+\frac{\partial \eta}{\partial x}+h \frac{\partial h}{\partial x}=0 .
\end{gathered}
$$


Utilizing (2.11) and integrating the result then produces the desired result of equation (2.12). It is interesting to note that equations (2.11) and (2.12) show that $\eta<0$ and $u_{1}=-u_{2}$ whenever $h=1 / 2$.

The lower layer dynamics can then be written in terms of the two partial differential equations (2.9) and (2.10) where (2.9) can be expressed as

$$
\frac{\partial u_{2}}{\partial t}+\left(u_{2}+\frac{\partial \eta}{\partial u_{2}}\right) \frac{\partial u_{2}}{\partial x}+\left(1+\frac{\partial \eta}{\partial h}\right) \frac{\partial h}{\partial x}=0
$$

with $\eta$ given by (2.12). For later reference, we denote the weak stratification equations as (2.11) and (2.12) with $u_{2}$ replaced by $u$ and equations (2.10) and (2.14) written in conservation form as

$$
\frac{\partial}{\partial t}\left(\begin{array}{l}
u \\
h
\end{array}\right)+\frac{\partial}{\partial x}\left(\begin{array}{c}
\frac{1}{2} u^{2}+\eta+h \\
h u
\end{array}\right)=\mathbf{0}
$$

2.2. Shallow-Layer Models We can use our previous work as a guide in developing a scaling suitable for thin lower and upper layers. In dimensional variables, the zero mass and momentum flux conditions (2.11) and (2.12) can be expressed as

$$
(H-h) u_{1}+h u_{2}=0
$$

and

$$
\frac{h u_{2}^{2}}{H-h}+g \eta+\frac{g^{\prime} h^{2}}{2 H}=0 .
$$

We do not necessarily know that these relations hold exactly in the limit of interest but we will use them as a scaling guide under the assumption that the balances they represent are reasonable.

Suppose we assume that $h(x, t) \simeq h_{0}$ and take $D=O\left(H-h_{0}\right)$. Here, $D$ is not defined explicitly in terms of known variables, but rather as an equivalence class of relations to avoid choosing several different scales for the following analysis. Using this notation, we find that in order for the balances in (2.16) and (2.17) to hold we must have

$$
\eta=O\left(\frac{g^{\prime} h_{0}^{2}}{g H}\right), \quad u_{2}=O\left(\sqrt{\frac{g^{\prime} D h_{0}}{H}}\right), \text { and } u_{1}=O\left(\frac{h_{0}}{D} \sqrt{\frac{g^{\prime} D h_{0}}{H}}\right) .
$$

Then, from the lower-layer continuity equation (2.4), we have that the horizontal length and time scales must be in the relation

$$
L / T=O\left(\sqrt{\frac{g^{\prime} D h_{0}}{H}}\right) .
$$


Quantities that play a role in subsequent developments are the two fractional depth parameters defined by

$$
\delta \equiv \frac{D}{H} \text { and } \epsilon \equiv \frac{h_{0}}{H}
$$

Based on the above scaling we now nondimensionalize equations (2.1) to (2.4) according to the new variables

$$
h=h_{0} \widetilde{h}, \quad \eta=\left(\frac{g^{\prime} h_{0}^{2}}{g H}\right) \tilde{\eta}, \quad u_{1}=\frac{h_{0}}{D} \sqrt{\frac{g^{\prime} D h_{0}}{H}} \widetilde{u}_{1}, \quad u_{2}=\sqrt{\frac{g^{\prime} D h_{0}}{H}} \widetilde{u}_{2} .
$$

The equations of motion are then found by substituting (2.21) into (2.1) to (2.4) and dropping the tildes. These are

$$
\begin{gathered}
\delta \frac{\partial u_{1}}{\partial t}+\epsilon u_{1} \frac{\partial u_{1}}{\partial x}+\delta \frac{\partial \eta}{\partial x}=0 \\
\delta \frac{\partial}{\partial t}\left[\frac{g^{\prime}}{g} \epsilon \eta-h\right]+\frac{\partial}{\partial x}\left[\left(1+\frac{g^{\prime}}{g} \epsilon^{2} \eta-\epsilon h\right) u_{1}\right]=0 \\
\delta \frac{\partial u_{2}}{\partial t}+\delta u_{2} \frac{\partial u_{2}}{\partial x}+\epsilon \frac{\partial \eta}{\partial x}+\frac{\partial h}{\partial x}-\epsilon \frac{g^{\prime}}{g} \frac{\partial \eta}{\partial x}=0
\end{gathered}
$$

and

$$
\frac{\partial h}{\partial t}+\frac{\partial}{\partial x}\left(h u_{2}\right)=0
$$

System (2.22) to (2.25) has three parameters, $\delta, \epsilon$, and $g^{\prime} / g$. The weakly stratified model of Subsection 2.1 is recovered by taking $\delta \simeq \epsilon=O(1)$ and $0<g^{\prime} / g \ll 1$. The leading-order equations for the thin lower layer are obtained from (2.22) to (2.25), for $0<\epsilon \ll 1, \delta=O(1)$, and are

$$
\frac{\partial u_{1}}{\partial t}+\frac{\partial \eta}{\partial x}=0, \quad \frac{\partial h}{\partial t}-\frac{\partial u_{1}}{\partial x}=0,
$$

and

$$
\frac{\partial u_{2}}{\partial t}+u_{2} \frac{\partial u_{2}}{\partial x}+\frac{\partial h}{\partial x}=0, \quad \frac{\partial h}{\partial t}+\frac{\partial}{\partial x}\left(h u_{2}\right)=0 .
$$

Equations (2.26) determine the motion of the upper layer and are linear whereas equations (2.27) for the thin lower layer are fully nonlinear. This is to be expected as linear momentum is to be (at least approximately) conserved so that only comparatively small velocities will be generated in the "thick" upper layer by the motion of the "thin" 
dense bottom layer. The complete dynamics in this case can then be resolved by first solving the system (2.27) written in conservation form as

$$
\frac{\partial}{\partial t}\left(\begin{array}{c}
u_{2} \\
h
\end{array}\right)+\frac{\partial}{\partial x}\left(\begin{array}{c}
\frac{1}{2} u_{2}^{2}+h \\
h u_{2}
\end{array}\right)=\mathbf{0}
$$

and using these results in

$$
u_{1}=-h u_{2}, \quad \eta=-\frac{1}{2} h^{2}-h u_{2}^{2}
$$

For a thin upper layer, the balances (2.22) to (2.25) suggest a rescaling of our variables according to

$$
h=1-\delta \tilde{h}, \quad \eta=\delta \tilde{\eta}, \quad\left(u_{1}, u_{2}\right)=\delta\left(\tilde{u}_{1}, \tilde{u}_{2}\right) .
$$

As before, when the transformation (2.30) is substituted into (2.22) to (2.25) and the tilde notation suppressed, the leading order equations for $\epsilon=O(1)$ and $0<\delta \ll 1$ become

$$
\begin{gathered}
\frac{\partial u_{1}}{\partial t}+u_{1} \frac{\partial u_{1}}{\partial x}+\frac{\partial \eta}{\partial x}=0 \\
\frac{\partial}{\partial t}\left[\frac{g^{\prime}}{g} \eta+h\right]+\frac{\partial}{\partial x}\left[\left(\frac{g^{\prime}}{g} \eta+h\right) u_{1}\right]=0
\end{gathered}
$$

and

$$
\frac{\partial \eta}{\partial x}-\frac{\partial h}{\partial x}-\frac{g^{\prime}}{g} \frac{\partial \eta}{\partial x}=0, \quad-\frac{\partial h}{\partial t}+\frac{\partial u_{2}}{\partial x}=0
$$

As seen for the case of the thin-lower layer equations, we have a nonlinear system (2.31) for the layer of interest written in conservation form as

$$
\frac{\partial}{\partial t}\left(\begin{array}{c}
u_{1} \\
\eta
\end{array}\right)+\frac{\partial}{\partial x}\left(\begin{array}{c}
\frac{1}{2} u_{1}^{2}+\eta \\
\eta u_{1}
\end{array}\right)=\mathbf{0}
$$

together with the algebraic relations

$$
h=\left(1-\frac{g^{\prime}}{g}\right) \eta \quad \text { and } \quad u_{2}=-\left(1-\frac{g^{\prime}}{g}\right) \eta u_{1}
$$

for determining the lower-layer dynamics. 


\section{Numerical Results}

Numerical results for the various systems in Section 2 are achieved using a relaxation scheme for conservation laws as described in [8]. The scheme approximates a given system of conservation laws with a nonhomogeneous linear hyperbolic system containing a small dissipative correction. The main advantage of this method is the straightforward application to an arbitrary system of conservation laws. The relaxation schemes are formally valid for flux vectors $f$ which may not be strictly hyperbolic or of mixed type. Second-order accuracy is achieved along with convergence to the correct weak solutions and the removal of oscillations at the trailing or leading edge of a shock [8].

For a one-dimensional general system of conservation laws,

$$
\frac{\partial \mathbf{u}}{\partial t}+\frac{\partial \mathbf{f}(\mathbf{u})}{\partial \dot{x}}=\mathbf{0}, \quad(x, t) \in \mathbb{R} \times \mathbb{R}^{+}, \mathbf{u} \in \mathbb{R}^{n}, \mathbf{f}(\mathbf{u}) \in \mathbb{R}^{n},
$$

the relaxation system to be solved is defined as

$$
\frac{\partial \mathbf{u}}{\partial t}+\frac{\partial \mathbf{v}}{\partial x}=\mathbf{0}, \quad \mathbf{v} \in \mathbb{R}^{n}, \quad \frac{\partial \mathbf{v}}{\partial t}+\mathbf{A} \frac{\partial \mathbf{u}}{\partial x}=-\frac{1}{\varepsilon}(\mathbf{v}-\mathbf{f}(\mathbf{u})),
$$

where $0<\varepsilon$ is a small constant and $\mathbf{A}=a \mathbf{I}$ is a diagonal matrix such that $0<a \in \mathbb{R}$. The system is dissipative provided that the condition

$$
\lambda^{2}<a, \quad \lambda=\max _{1 \leq i \leq n}\left|\lambda_{i}(\mathbf{u})\right|
$$

holds, where $\lambda_{i}(\mathbf{u})$ is an eigenvalue of the Jacobian matrix $\mathbf{f}^{\prime}(\mathbf{u})$.

Here the numerical method used is a second-order total variation-diminishing Runge-Kutta splitting scheme with Van Leer's slope limiter [11] to solve (3.2). This scheme has the correct zero relaxation limit so that as $\varepsilon \rightarrow 0$ this discretization becomes a second-order approximation to the original system (3.1). The largest difficulty with this numerical scheme can now be seen as the predetermination of the constant matrix $\mathbf{A}$ and its effect on determining the numerical grid sizes to satisfy the CFL condition

$$
\sqrt{a} \frac{k}{h} \leq 1,
$$

where $k$ is the uniform time step and $h$ is the uniform grid width for the spatial variable $x$. Ideally, the constant $a$ should be chosen as small as possible while satisfying (3.3) to minimize computing time. For the calculations, a uniform spatial grid width of $h=0.01$ was used. The parameter $a$ was chosen to satisfy (3.3) and the uniform time step $k$ then picked according to (3.4) with CFL number approximately 0.5. For all calculations, $\varepsilon=10^{-11}$ or $10^{-10}$ and the difference here was inconsequential. 


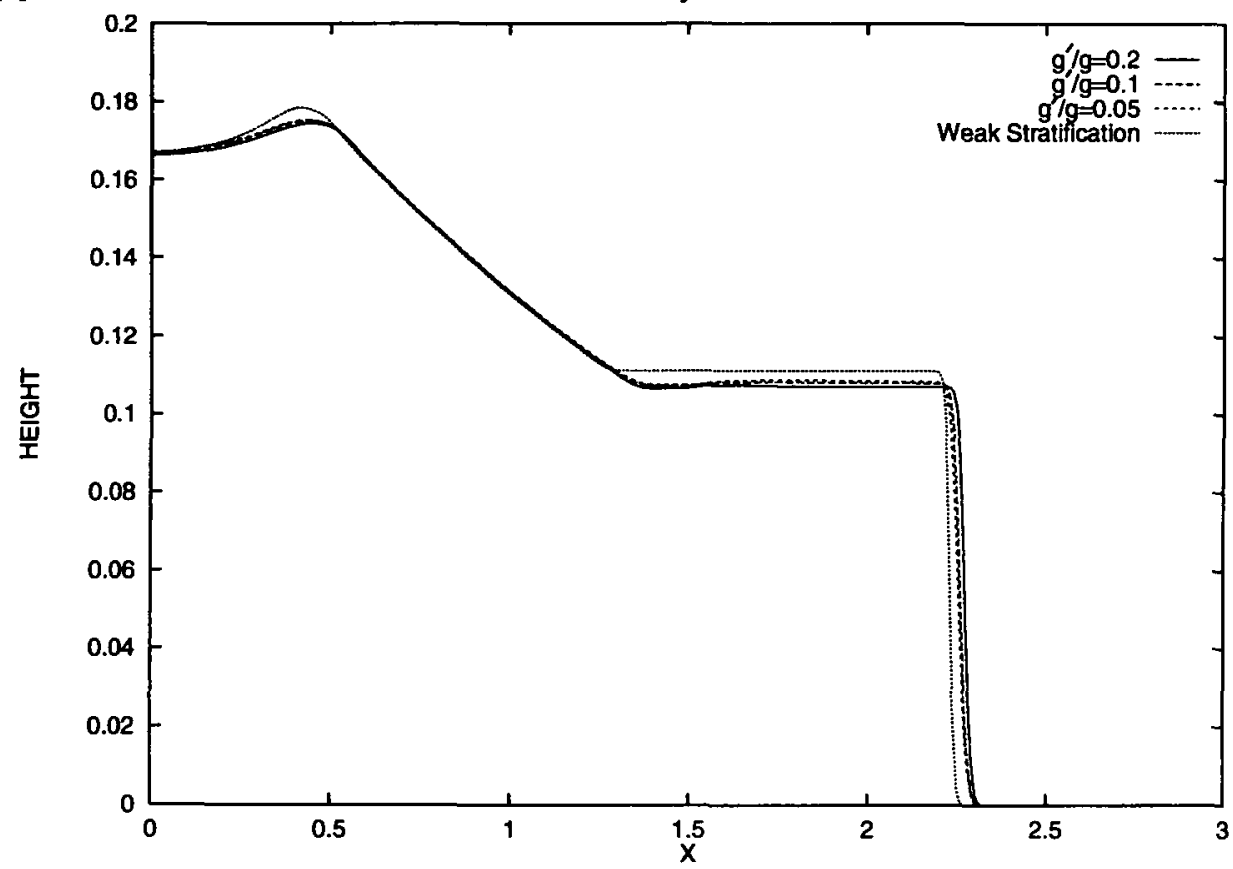

FIGURE 2A. Comparison of solutions to the full equations (2.6) with solutions to the weak-stratification equations (2.11) at non-dimensional time 3 for $h_{0}=0.3$.

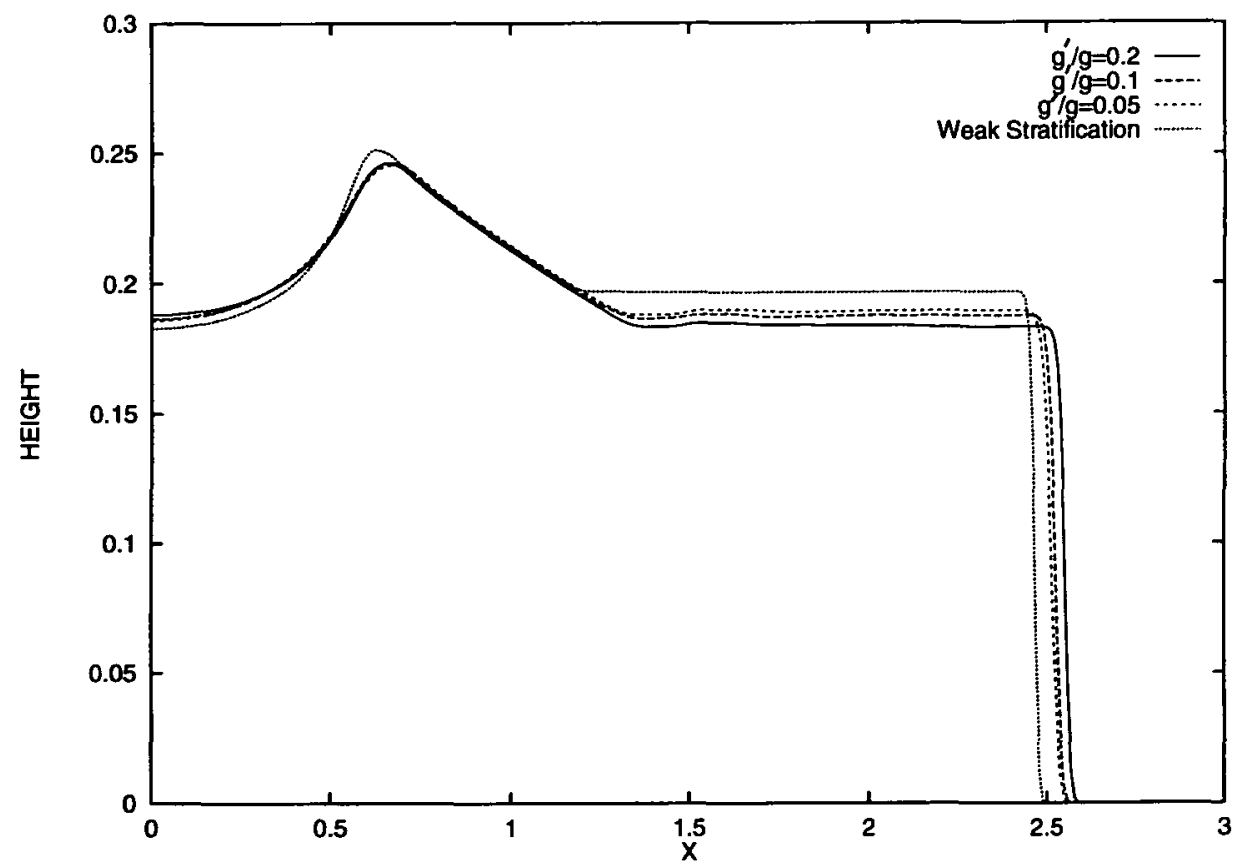

FIGURE 2B. Comparison of solutions to the full equations (2.6) with solutions to the weak-stratification equations (2.11) at non-dimensional time 3 for $h_{0}=0.5$. 


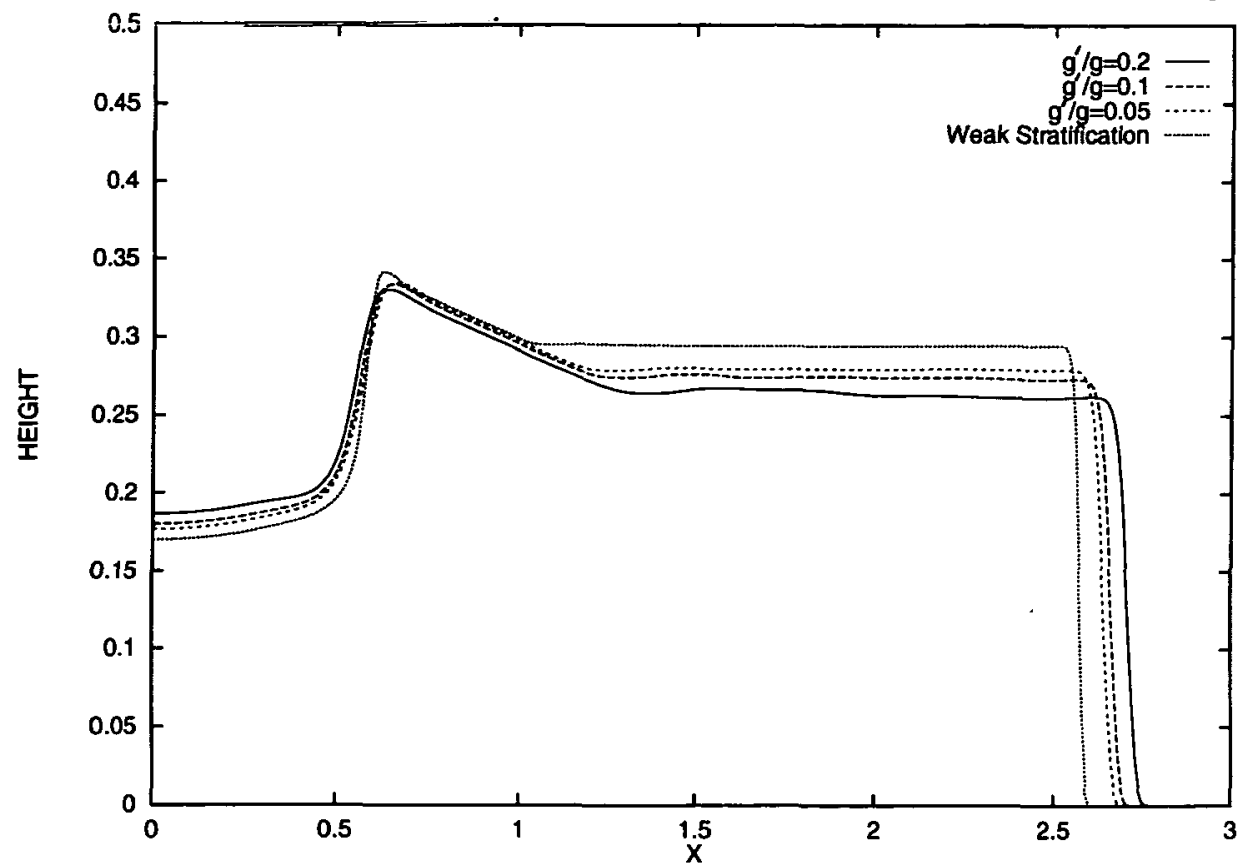

FIGURE 2C. Comparison of solutions to the full equations (2.6) with solutions to the weak-stratification equations (2.11) at nondimensional time 3 for $h_{0}=0.7$.

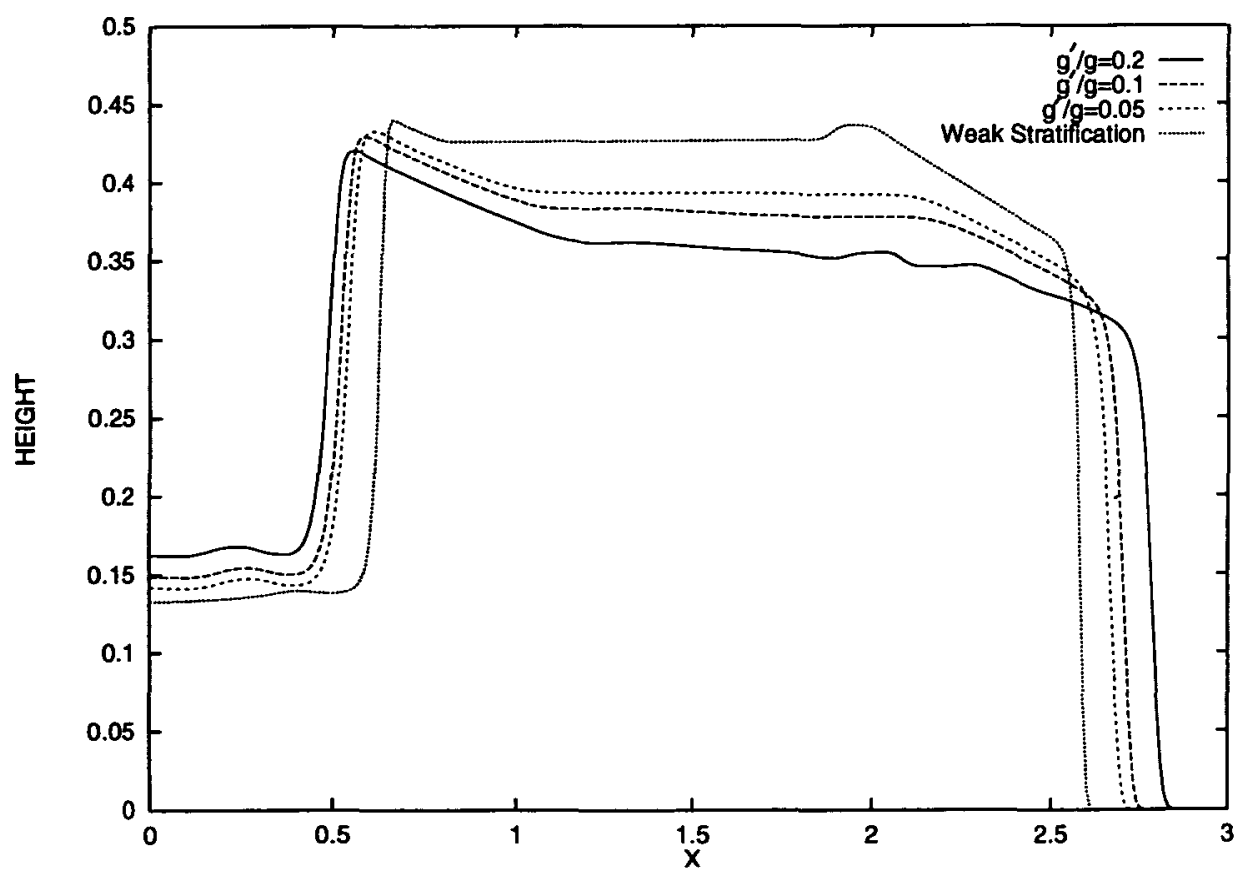

FIGURE 2D. Comparison of solutions to the full equations (2.6) with solutions to the weak-stratification equations (2.11) at nondimensional time 3 for $h_{0}=0.9$. 
For the numerical calculations the problem to be considered in the remainder of this section is that of the sudden release of a rectangular shaped area of fluid at rest into a half-space region of less dense undisturbed fluid. The boundary conditions consist of a rigid wall at the left $(x=0)$ and undisturbed fluid in the semi-infinite region to the right.

The initial conditions are implemented for a constant value $x_{0}$ as

$$
\begin{gathered}
u_{1}(x, 0)=0, \quad u_{2}(x, 0)=0, \quad \eta(x, 0)=0, \\
h(x, 0)= \begin{cases}h_{0} & \text { for } x \leq x_{0}, \\
0 & \text { for } x>x_{0},\end{cases}
\end{gathered}
$$

and for all calculations, $x_{0}$ was chosen to be the constant 1 .

The boundary conditions at the wall $x=0$ can be determined from the governing equations (2.1) and are written for $t>0$ as

$$
\begin{gathered}
u_{1}(0, t)=0, \quad u_{2}(0, t)=0, \\
\frac{\partial \eta}{\partial x}(0, t)=0, \text { and } \frac{\partial h}{\partial x}(0, t)=0 .
\end{gathered}
$$

Boundary conditions for $\mathbf{v}$ are determined $[8]$ as $\mathbf{v}(x, 0)=\mathbf{f}(\mathbf{u}(x, 0))$, with the corresponding initial conditions given by $\mathbf{v}(0, t)=\mathbf{f}(\mathbf{u}(0, t))$ or the gradient conditions $\frac{\partial \mathbf{v}}{\partial x}(0, t)=\mathbf{f}^{\prime}(\mathbf{u}(0, t)) \frac{\partial \mathbf{u}}{\partial x}(0, t)$ as applicable.

To investigate the region of validity of the weak-stratification model and provide a check on the numerical scheme, numerical solutions to (2.6) are plotted for various values of $g^{\prime} / g$ in Figure 2 along with solutions to (2.15). For convenience, equations (2.6) are referred to as the full equations and (2.15) as the weak-stratification equations. The interface height profiles $h(x, t)$ are shown at a single time since these are the most easy to compare quantities.

Figures 2A to 2D are in good agreement with results produced in [3] by another numerical method. As expected, solutions of the full equations approach solutions of the weak stratification equations as $g^{\prime} / g \rightarrow 0$. For Figures $2 \mathrm{~A}$ and $2 \mathrm{~B}$, the gravity current quickly slumps from the initial state (3.6) to a curve which begins to take the form of the similarity solution. For Figures $2 \mathrm{C}$ and 2D, rear shocks have formed. This difference in the interface height is due to the increased initial values $h_{0}=0.7,0.9$, and the high backflow velocity $u_{1}$ which may interact with the endwall to produce this effect [3]. For $h_{0} \leq 0.5$ this shock formation was not observed numerically, and this matter will be addressed in Section 5.

To investigate the thin lower-layer model, solutions to the thin lower-layer equations (2.28) are plotted in Figure 3 along with solutions to the full equations for varying values of $h_{0}$. The calculations are performed with $a=75$ for the full equations and 


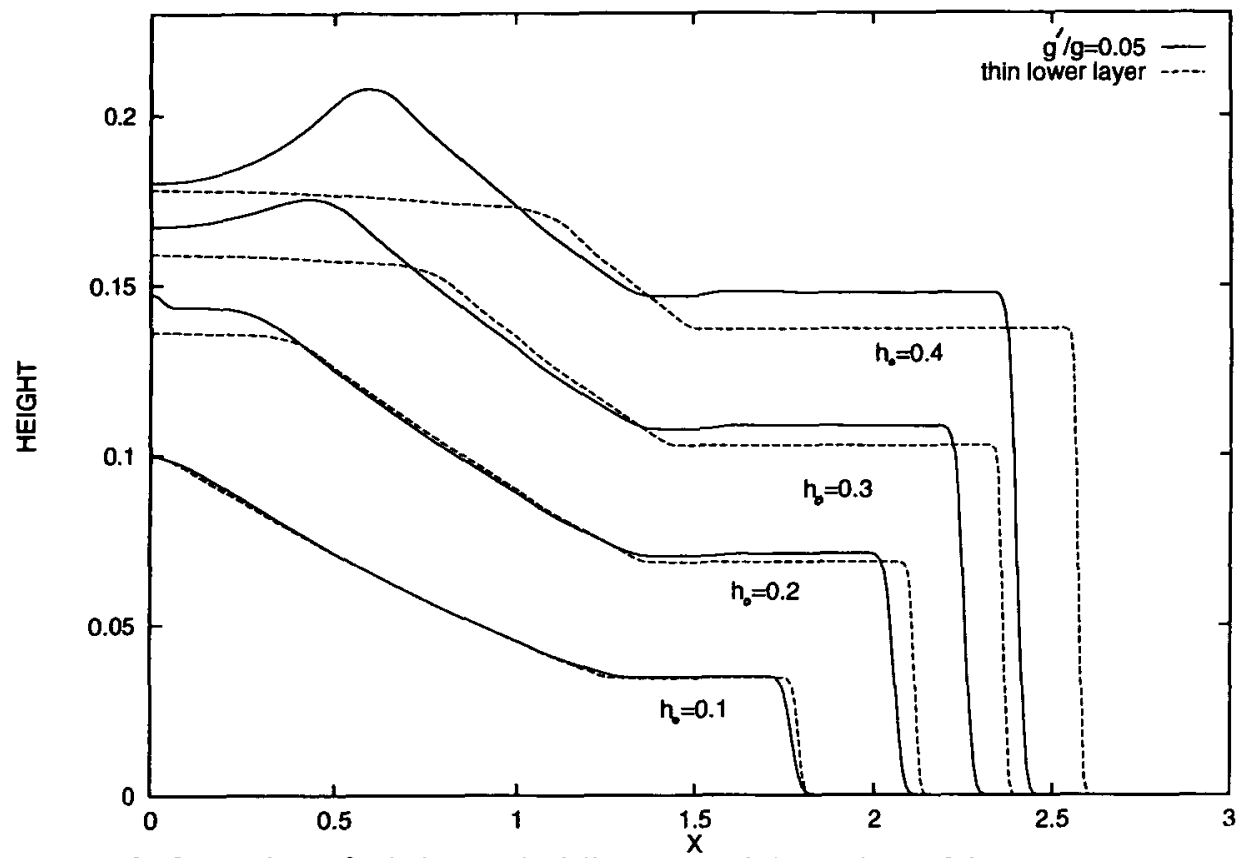

FIGURE 3. Comparison of solutions to the full equations (2.6) for $g^{\prime} / g=0.05$ with solutions to the thin lower-layer equations (2.24) at nondimensional time 3 for $h_{0}=0.1,0.2,0.3$ and 0.4 .

$a=4$ for the thin lower layer. As expected, the agreement is good for the thin lower-layer region where $h_{0} \leq 0.2$.

For larger values of $h_{0}$ the solutions become different in character, as the thin lower-layer equations do not capture the rear shock, which develops in the same way as for the weakly stratified case in Figures 2C and 2D. As seen in Figure 4, this disagreement is more pronounced for $h_{0} \geq 0.5$, which is expected since the upper layer begins to interact with the lower layer and the endwall, as $h_{0}$ increases, thus removing the assumption that the layers are essentially decoupled. The importance of $h_{0}=0.5$ is investigated further in Section 5 .

To examine solutions to the thin upper-layer equations (2.33) the scaling (2.30) must be taken into account in the initial conditions (3.5) and (3.6). Here, the initial condition used is the sudden release of a rectangular block of light fluid into an undisturbed region of heavier fluid. This is given by equations (3.5) with the initial interface height given by

$$
h(x, 0)=\left\{\begin{array}{rll}
h_{0} & \text { for } & x \leq x_{0} \\
1 & \text { for } & x>x_{0}
\end{array}\right.
$$

The boundary conditions (3.7) are unchanged.

If the parameter $\delta$ is fixed, the scaling (2.30) suggests that the initial condition for 


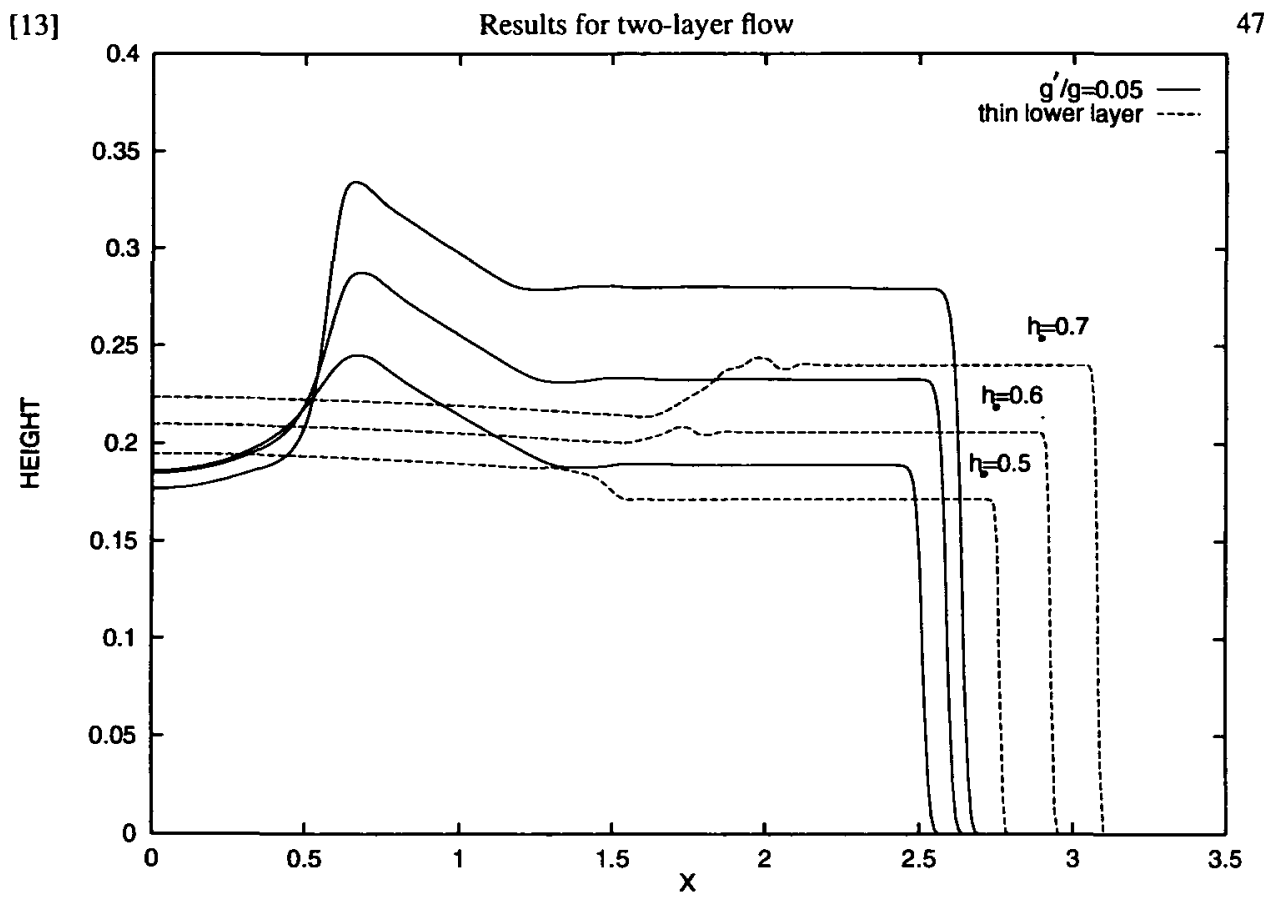

FIGURE 4. Comparison of solutions to the full equations (2.6) for $g^{\prime} / g=0.05$ with solutions to the thin lower-layer equations (2.24) at nondimensional time 3 for $h_{0}=0.5,0.6$ and 0.7 .

the thin upper-layer variable $\widetilde{h}$ is given by

$$
\tilde{h}(x, 0)=\left\{\begin{array}{rll}
\tilde{h}_{0} & \text { for } & x \leq x_{0} \\
0 & \text { for } & x>x_{0}
\end{array}\right.
$$

In (3.9) $\widetilde{h}_{0} \equiv\left(1-h_{0}\right) / \delta$. Using (2.34) in (2.33) gives the thin upper-layer equations written without the tildes as

$$
\frac{\partial}{\partial t}\left(\begin{array}{c}
u_{1} \\
h
\end{array}\right)+\frac{\partial}{\partial x}\left(\begin{array}{c}
\frac{1}{2} u_{1}^{2}+\rho_{1} h / \rho_{2} \\
h u_{1}
\end{array}\right)=\mathbf{0}
$$

Solutions to these equations are plotted in Figures $5 \mathrm{~A}$ to $5 \mathrm{C}$ for various values of $g^{\prime} / g$ and pre-scaled initial height $\widetilde{h}_{0}$. The interface height $1-\delta \widetilde{h}_{0}$ is shown and the calculations were done with $a=25$. The upper-layer gravity current slows down with increasing values of $g^{\prime} / g$ as more momentum is transferred to the lower layer. As well, the solutions with the lowest value of $g^{\prime} / g$ in Figure 5A approach the similarity solution profile in the shortest time.

As a check on the numerical solutions of the thin-layer equations, the numerical solutions are compared to the similarity solutions described in [3] and [7]. For the 
thin lower layer the similarity solution is given by

$$
\begin{gathered}
x_{f}=3\left(\frac{h_{0} x_{0}}{4}\right)^{1 / 3} t^{2 / 3}, \\
u_{2}=\left\{\begin{array}{ll}
\frac{2}{3} \frac{x}{t} & \text { for } x \leq x_{f}, \\
0 & \text { for } x>x_{f},
\end{array} \quad h= \begin{cases}\frac{x^{2}+x_{f}^{2}}{9 t^{2}} & \text { for } x \leq x_{f} \\
0 & \text { for } x>x_{f},\end{cases} \right.
\end{gathered}
$$

where $x_{f}$ is the front position. The thin upper-layer equations also have a similarity solution for the initial condition (3.9) which is given using the thin upper-layer variables by

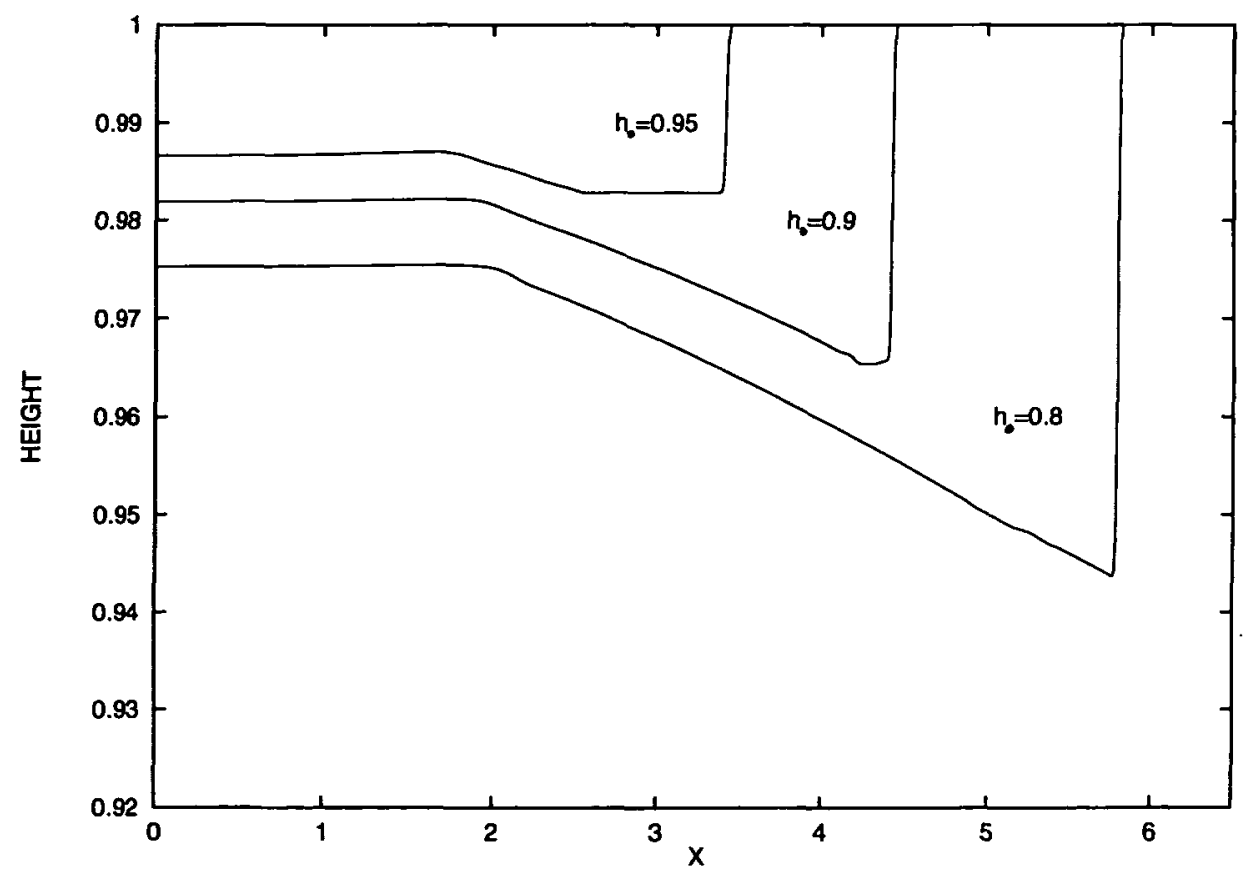

FIGURE 5A. Solutions to the thin upper-layer equations (3.10) at nondimensional time 3 for $h_{0}=$ $0.8,0.9,0.95$ with $g^{\prime} / g=0.05$. 


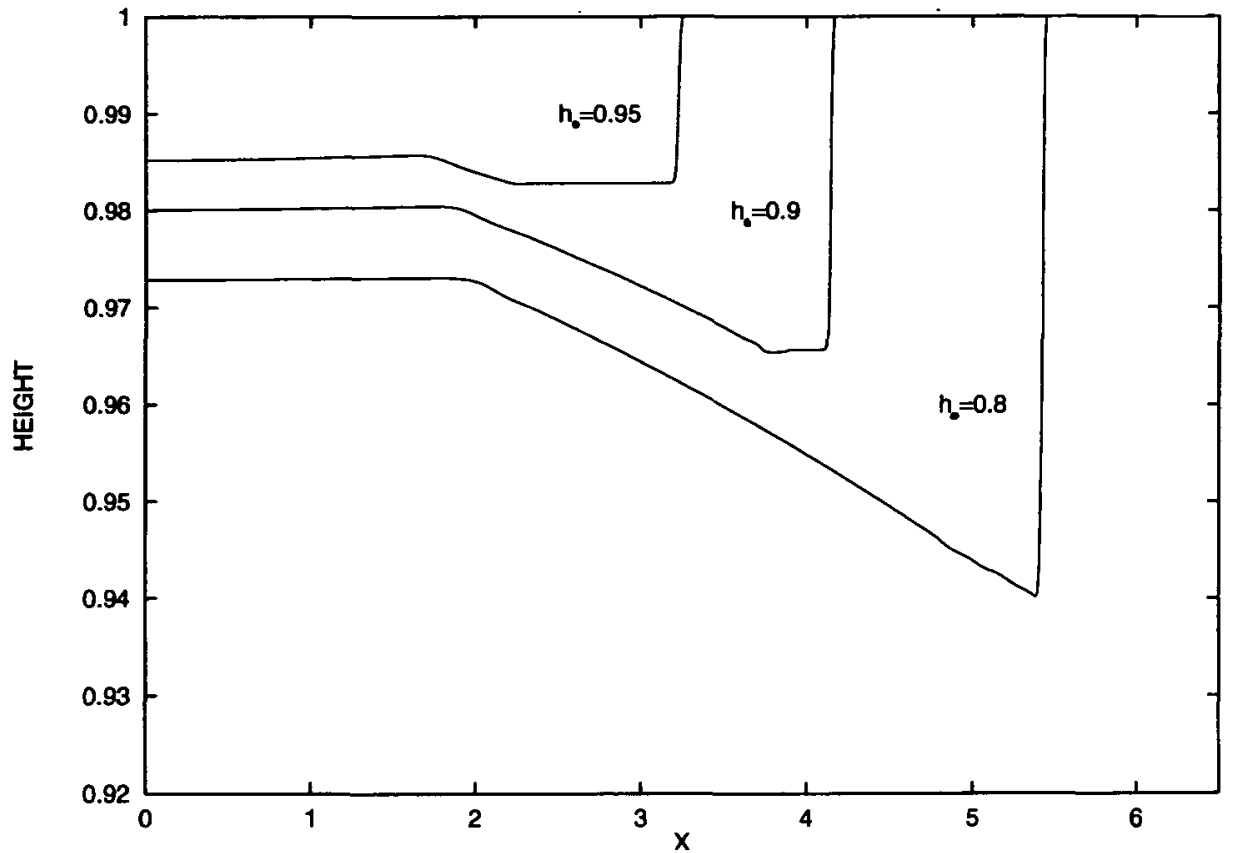

FIGURE 5B. Solutions to the thin upper-layer equations (3.10) at nondimensional time 3 for $h_{0}=$ $0.8,0.9,0.95$ with $g^{\prime} / g=0.2$.

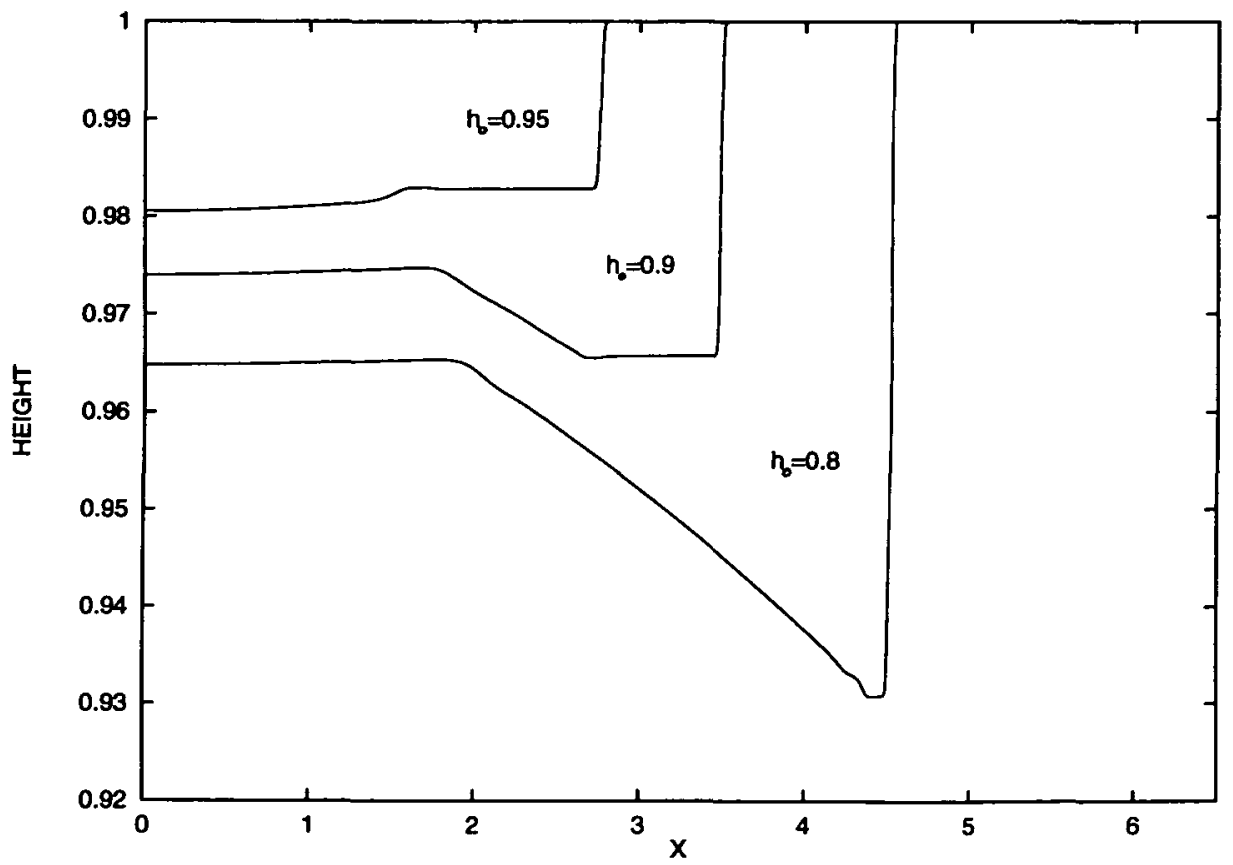

FIGURE 5C. Solutions to the thin upper-layer equations (3.10) at nondimensional time 3 for $h_{0}=$ $0.8,0.9,0.95$ with $g^{\prime} / g=0.5$. 


$$
\begin{gathered}
x_{f}=3\left(\frac{\rho_{1} \tilde{h}_{0} x_{0}}{4 \rho_{2}}\right)^{1 / 3} t^{2 / 3}, \\
u_{2}=\left\{\begin{array}{ll}
\frac{2}{3} \frac{x}{t} & \text { for } x \leq x_{f}, \\
0 & \text { for } x>x_{f},
\end{array} \quad h= \begin{cases}\frac{\rho_{1}}{\rho_{2}}\left(\frac{x^{2}+x_{f}^{2}}{9 t^{2}}\right) & \text { for } x \leq x_{f}, \\
0 & \text { for } x>x_{f} .\end{cases} \right.
\end{gathered}
$$

Some similarity solutions for the interface height $h$ are plotted using the thin-layer variables (2.21), in Figures 6A and 6B with $x_{0}=1$. Although at first glance these seem to indicate a large variation between the solutions, the graphing scale used is such that the actual disagreement is less than $0.3 \%$ of the total scaled height of the two layers.

Another check on the numerics is given by the speed of the leading discontinuity or shock which can be calculated via the Rankine-Hugoniot jump conditions [15]. For a discontinuity propagating with speed $d$, these jump conditions for a conservation law of the form (3.1) are given by

$$
\mathbf{f}\left(\mathbf{u}^{+}\right)-\mathbf{f}\left(\mathbf{u}^{-}\right)=d\left(\mathbf{u}^{+}-\mathbf{u}^{-}\right)
$$

where $\mathbf{u}^{+}, \mathbf{u}^{-}$are the constant values on the right and left sides respectively of the discontinuity. For the full equations (2.6) the initial conditions (3.5) and (3.6) give these constants as

$$
\mathbf{u}^{+}=\left(\begin{array}{l}
0 \\
0 \\
0 \\
0
\end{array}\right), \quad \mathbf{u}^{-}=\left(\begin{array}{c}
u_{1}^{-} \\
\eta^{-} \\
u_{2}^{-} \\
h^{-}
\end{array}\right) .
$$

Condition (3.13) is then a system of 4 equations in the 5 unknowns $d, u_{1}^{-}, \eta^{-}, u_{2}^{-}$, and $h^{-}$. However, the fourth equation is simply $h^{-} u_{2}^{-}=d h^{-}$so that for nonzero $h^{-}$the shock speed is given by $d=u_{2}^{-}$. This condition is easily verifiable numerically, and was found to be in good agreement with the computed values of $u_{2}^{-}$.

For the three-model systems in conservation form, the condition (3.13) can give a relation for the shock speed and the interface height. For example, equation (2.28) for the thin lower layer gives $d=\sqrt{2 h^{-}}$while for the weak-stratification equations (2.15), we have

$$
d^{2}=\frac{1-h^{-}}{1+h^{-}}\left(2 h^{-}-h^{-2}\right)<2 h^{-},
$$

which concurs with the difference in shock speeds seen in Figures 3 and 4. 


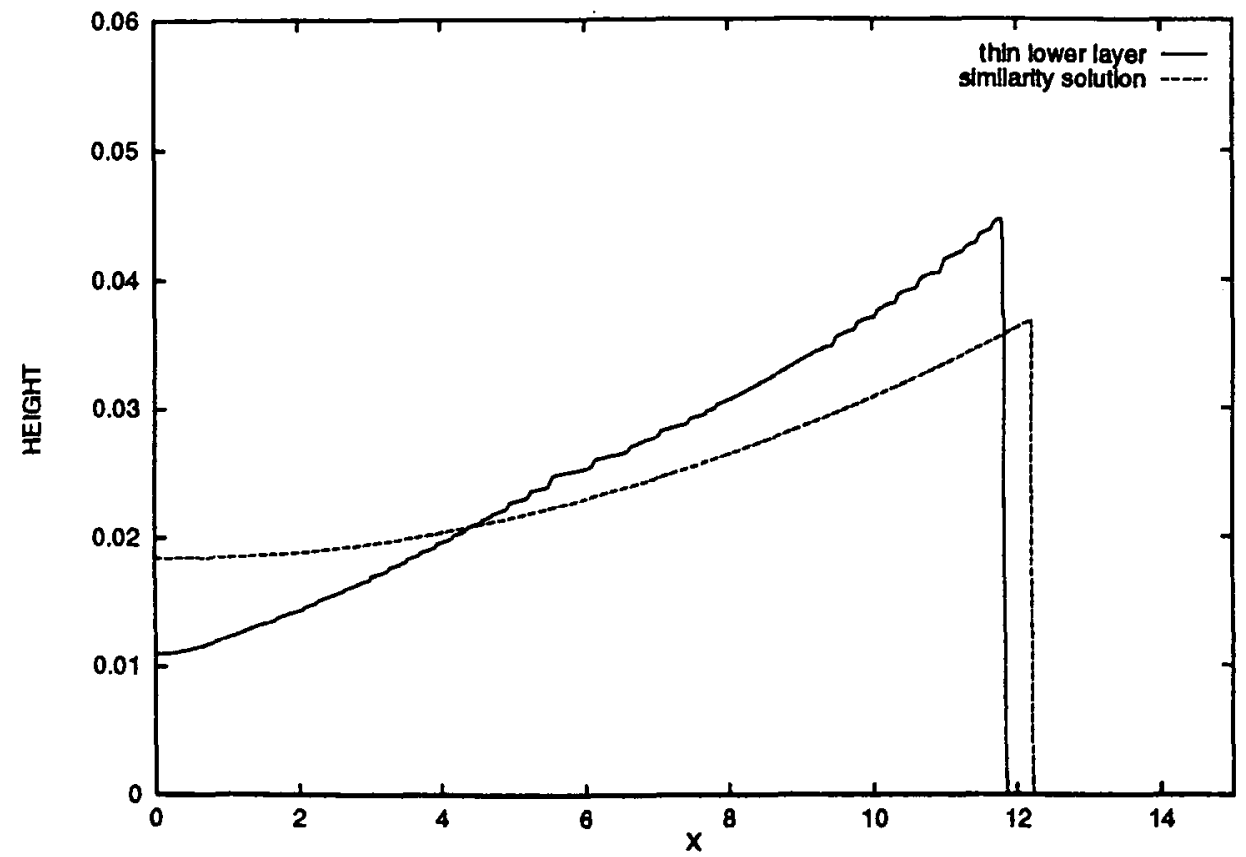

FIGURE 6A. Numerical and similarity solutions to the thin lower-layer equations (2.24) at nondimensional time 30 for $h_{0}=0.2$.

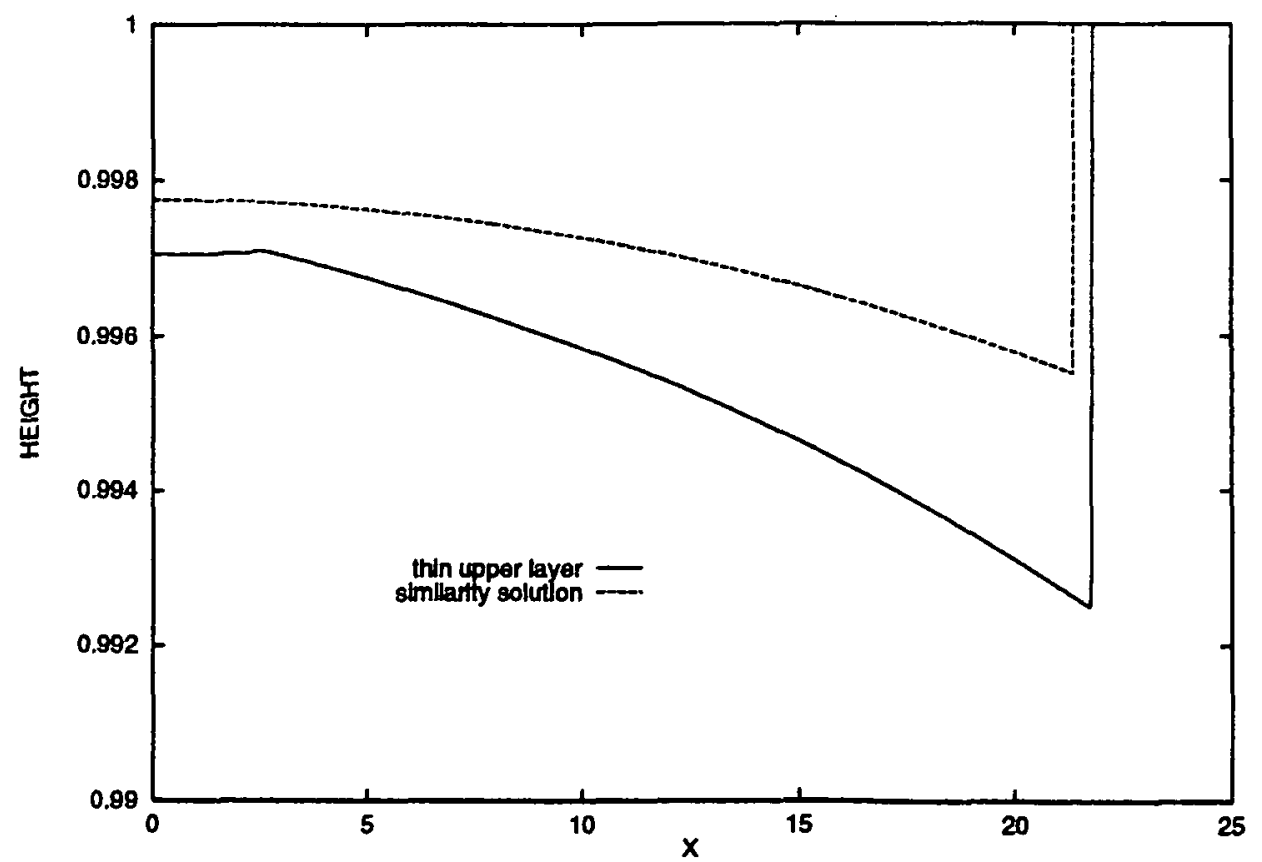

FIGURE 6B. Numerical and similarity solutions to the thin upper-layer equations (3.10) at nondimensional time 30 for $h_{0}=0.9$ and $g^{\prime} / g=0.2$. 


\section{Hyperbolicity}

All of our model systems can be written in the conservation form

$$
\frac{\partial \mathbf{u}}{\partial t}+\frac{\partial \mathbf{f}(\mathbf{u})}{\partial x}=\mathbf{0}
$$

by defining appropriate vectors $\mathbf{u}$ of the dependent variables and the vector-valued flux functions $\mathbf{f}: \mathbb{R}^{2} \rightarrow \mathbb{R}^{2}$ for each of the systems examined in Section 2 . For the weakly stratified, thin lower-layer, and thin upper-layer models, the corresponding state vectors are

$$
\mathbf{u} \equiv\left[\begin{array}{c}
u \\
h
\end{array}\right], \quad \mathbf{u} \equiv\left[\begin{array}{c}
u_{2} \\
h
\end{array}\right], \quad \text { and } \quad \mathbf{u} \equiv\left[\begin{array}{c}
u_{1} \\
h
\end{array}\right],
$$

respectively. The associated flux functions for (4.2) are

$$
\mathbf{f} \equiv\left[\begin{array}{c}
\frac{u^{2}}{2}+h-\frac{h^{2}}{2}-\frac{h u^{2}}{1-h} \\
h u
\end{array}\right], \mathbf{f} \equiv\left[\begin{array}{c}
\frac{u_{2}^{2}}{2}+h \\
h u_{2}
\end{array}\right], \text { and } \mathbf{f} \equiv\left[\begin{array}{c}
\frac{u_{1}^{2}}{2}+\eta \\
\eta u_{1}
\end{array}\right] .
$$

The two conservation equations for the thin-layer models are strictly hyperbolic for all physical values of the state variables. This is not the case for the weakly-stratified equations. The eigenvalues associated with (4.1) for the weakly stratified case are $\lambda^{ \pm}$, where

$$
\lambda^{ \pm}=\frac{u(1-2 h)}{1-h} \pm \frac{1}{1-h} \sqrt{h(1-h)\left[(1-h)^{2}-u^{2}\right]} .
$$

The system is hyperbolic where $\lambda^{ \pm}$are real. This region of hyperbolicity, labeled $S$, is shown in Figure 7.

We shall show that this region is invariant [6] in the sense that a vector $\mathbf{u}(x, t)$ satisfying the Cauchy problem (4.1) with the initial condition $\mathbf{u}(x, 0)=\mathbf{u}_{0}(x)$ has the property that $\mathbf{u}(x, t) \in S$ for all $(x, t)$ whenever $\mathbf{u}(x, 0)=\mathbf{u}_{0} \in S$.

Associated with the eigenvalues $\lambda^{ \pm}$of (4.4) are the left and right eigenvectors $I$ and $r$ given by

$$
\mathrm{I}^{ \pm}=\left[\frac{h(1-h)}{u h \pm \sqrt{h(1-h)\left[(1-h)^{2}-u^{2}\right]}}, 1\right]
$$

and

$$
\mathbf{r}^{ \pm}=\frac{(1-h)^{3}-u^{2}}{ \pm 2(1-h) \sqrt{h(1-h)\left[(1-h)^{2}-u^{2}\right]}}\left[\begin{array}{c}
1 \\
-u h \pm \sqrt{h(1-h)\left[(1-h)^{2}-u^{2}\right]}
\end{array}\right]
$$


where these have been chosen to satisfy the orthonormality conditions

$$
\mathbf{I}^{ \pm} \cdot \mathbf{r}^{\mp}=0, \quad \mathbf{I}^{ \pm} \cdot \mathbf{r}^{ \pm}=1 .
$$

It can be shown that the characteristic fields are genuinely nonlinear [5] in that

$$
\operatorname{grad}_{\mathbf{u}} \lambda^{ \pm} \cdot \mathbf{r}^{ \pm} \neq 0
$$

with the exception of a local linear degeneracy [5] about the state $\mathbf{u}=(0,1 / 2)$.

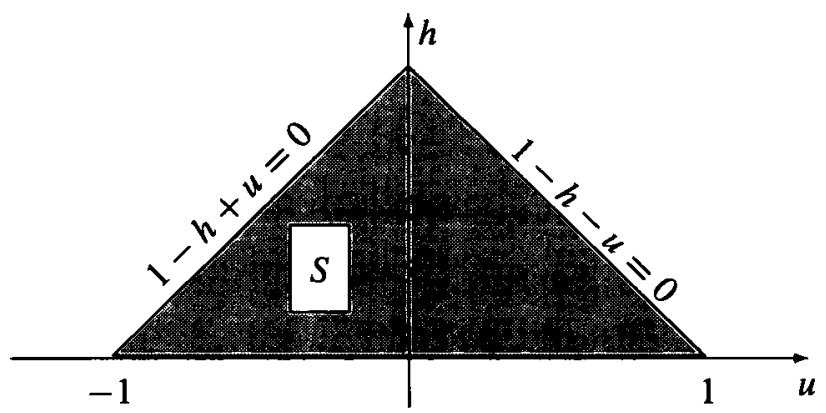

FIGURE 7. Region of hyperbolicity for the weakly-stratified model (2.11).

Now, using a result from Hoff [6], for a system of two conservation laws with genuinely nonlinear characteristic fields, we see that the region $S$ in Figure 7 is invariant if and only if $S$ is convex and the normal to $\partial S$ is parallel to a left eigenvector of $\mathbf{f}^{\prime}(\mathbf{u})$ at each point of $\partial S$. Here the boundary $\partial S$ consists of three straight line segments labeled with subscripts and defined as

$$
\partial S_{1}=\{(u, 0):-1 \leq u \leq 1\}, \quad \partial S_{2}=\{(h, 1-h): 0<h \leq 1\}
$$

and

$$
\partial S_{3}=\{(h, h-1): 0<h<1\} .
$$

The outward normal vectors and left eigenvectors on these segments can be calculated as

$$
\begin{array}{cc}
l & l \\
\left.\mathbf{n}\right|_{\partial S_{1}}=[0,-1], & \left.\mathbf{I}^{ \pm}\right|_{\partial S_{1}}=[0,1], \\
\left.\mathbf{n}\right|_{\partial S_{2}}=2^{-1 / 2}[1,1], & \left.\mathbf{I}^{ \pm}\right|_{\partial S_{2}}=[1,1], \\
\left.\mathbf{n}\right|_{\partial S_{3}}=2^{-1 / 2}[-1,-1], & \left.\mathbf{I}^{ \pm}\right|_{\partial S_{3}}=[-1,1] .
\end{array}
$$


In this case, it is clear that $\left.\mathbf{n}\right|_{\partial s_{i}}$ is parallel to $\left.\mathrm{I}^{ \pm}\right|_{\partial S_{i}}$ for $i=1,2,3$ so that the region of hyperbolicity $S$ is invariant.

The hyperbolicity of the equations can also be examined via the stability Froude number of the flow [10]. This is given for the dimensional equations (2.1) to (2.4) as

$$
F^{2}=\frac{\left(u_{2}-u_{1}\right)^{2}}{g^{\prime}(H+\eta)} .
$$

The solution to equations (2.1) to (2.4) is said to subcritical if $F^{2}<F_{\text {crit }}^{2}$ where $F_{\text {crit }}^{2}$ is a function of the variables $g^{\prime} / g, H, h, \eta$ and takes on values in the interval $1 \leq F_{\text {crit }}^{2} \leq 2$. For the weak stratification case $F_{\text {crit }}^{2} \simeq 1$ [10], so that the subcritical flow here is defined by the condition $F^{2} \leq 1$.

In the nondimensional weak-stratification variables (2.5) with $u_{2}$ replaced by $u$, (4.12) becomes

$$
F^{2}=\frac{\left(u-u_{1}\right)^{2}}{1+g^{\prime} \eta / g} .
$$

Using the algebraic conditions (2.11) and (2.12) for $u_{1}$ and $\eta$, the condition of subcritical flow can be expressed solely in terms of the lower-layer variables $u, h$ as

$$
u^{2}=(1-h)^{2}\left(1-\frac{g^{\prime}}{g} \frac{h^{2}}{2}\right)\left(1+\frac{g^{\prime}}{g} h(1-h)\right)^{-1} .
$$

In the weak stratification limit, for $g^{\prime} / g$ small, this result reduces to $u^{2} \leq(1-h)^{2}$ which is precisely the region of hyperbolicity depicted in Figure 7.

\section{Shock Formation}

From Section 3, the numerical solutions to the lower-layer and weak-stratification equations are not calculated at large enough times so that the similarity solutions are an accurate representation. For short times such as these, the interface sometimes contains a characteristic hump for large enough values of $h_{0}$, which moves to the right and catches up to the front of the gravity current. The method of weakly-nonlinear hyperbolic waves is used to examine the possibility that these smooth variations along the interface may form a shock in sufficient time to be observable numerically.

We examine the Cauchy problem (4.1) with initial data given by $\mathbf{u}(x, 0)=\mathbf{u}_{0}+$ $\varepsilon \hat{\mathbf{u}}(x)$ representing a perturbation from the constant hyperbolic solution $\mathbf{u}_{0}$ for a small positive parameter $\varepsilon$. For a vector $\mathbf{u}$ with two components, we look for a multiplescales expansion solution of the form [12]

$$
\mathbf{u}(x, t)=\mathbf{u}_{0}+\varepsilon\left[\sigma^{+}\left(\phi^{+}, \tau\right) \mathbf{r}^{+}+\sigma^{-}\left(\phi^{-}, \tau\right) \mathbf{r}^{-}\right]+O\left(\varepsilon^{2}\right),
$$


with the phase variables $\phi^{ \pm}=x-\lambda^{ \pm} t$, the slow time scale $\tau=\varepsilon t$, and $\lambda^{ \pm}$and $\mathbf{r}^{ \pm}$ the eigenvalues and right eigenvectors of $\mathbf{f}^{\prime}\left(\mathbf{u}_{0}\right)$.

The scalar functions $\sigma^{ \pm}$satisfy a decomposition of the initial condition given by

$$
\begin{aligned}
\hat{\mathbf{u}}(x) & =\sigma^{+}(x, 0) \mathbf{r}^{+}+\sigma^{-}(x, 0) \mathbf{r}^{-} \\
& \equiv \sigma_{0}^{+} \mathbf{r}^{+}+\sigma_{0}^{-} \mathbf{r}^{-} .
\end{aligned}
$$

The expansion is uniformly valid for time scales $O\left(\varepsilon^{-1}\right)$ if the two nonresonance conditions

$$
\dot{\sigma}^{ \pm}+\Gamma^{ \pm} \sigma^{ \pm} \sigma^{ \pm \prime}=0
$$

hold. In (5.3) the notation introduced is $\dot{\sigma}^{ \pm}=\partial \sigma^{ \pm} / \partial \tau$ and ${\sigma^{ \pm \prime}}^{\prime}=\partial \sigma^{ \pm} / \partial \phi^{ \pm}$. The constant terms $\Gamma^{ \pm}$are the interaction coefficients defined by

$$
\Gamma^{ \pm}=\mathbf{l}^{ \pm} \cdot \mathbf{B}\left(\mathbf{r}^{ \pm}, \mathbf{r}^{ \pm}\right)
$$

where, $\mathbf{l}^{ \pm}$are the left eigenvectors of $\mathbf{f}^{\prime}\left(\mathbf{u}_{0}\right)$ and $\mathbf{B}\left(\mathbf{r}^{ \pm}, \mathbf{r}^{ \pm}\right)$is a bilinear form found from the Taylor expansion of $\mathbf{f}$ via

$$
\mathbf{f}(\mathbf{u})=\mathbf{f}\left(\mathbf{u}_{0}\right)+\mathbf{f}^{\prime}\left(\mathbf{u}_{0}\right) \mathbf{u}+\frac{1}{2} \mathbf{B}(\mathbf{u}, \mathbf{u})+O\left(\|\mathbf{u}\|^{3}\right) .
$$

For the case of smooth initial data, (5.3) suggests that shocks will form in a finite time. The solutions are given implicitly [15] by

$$
\sigma^{ \pm}\left(\phi^{ \pm}, \tau\right)=\sigma_{0}^{ \pm}(s), \quad \phi^{ \pm}=s+\Gamma^{ \pm} \sigma_{0}^{ \pm}(s) \tau,
$$

which will be continuous until the breaking times $t_{B}$ which are given explicitly [15] by

$$
t_{B}=\min _{\xi} \frac{-1}{\Gamma^{ \pm} \sigma_{0}^{ \pm^{\prime}}(\xi)}
$$

Thus the signs of both $\Gamma^{ \pm}$and $\sigma_{0}^{ \pm \prime}$ are important in predicting whether or not shocks will form. This will be examined for the weakly-stratified equations and both the thin-layer equations.

For the weakly-stratified equations in the form (2.15), using $\mathbf{u}_{0}=\left(0, h_{0}\right)$ for $0<h_{0}<1$, a short calculation gives the interaction coefficients as

$$
\Gamma^{ \pm}= \pm \frac{3}{4} \sqrt{\frac{1-h_{0}}{h_{0}}}\left(\frac{1-2 h_{0}}{1-h_{0}}\right) .
$$




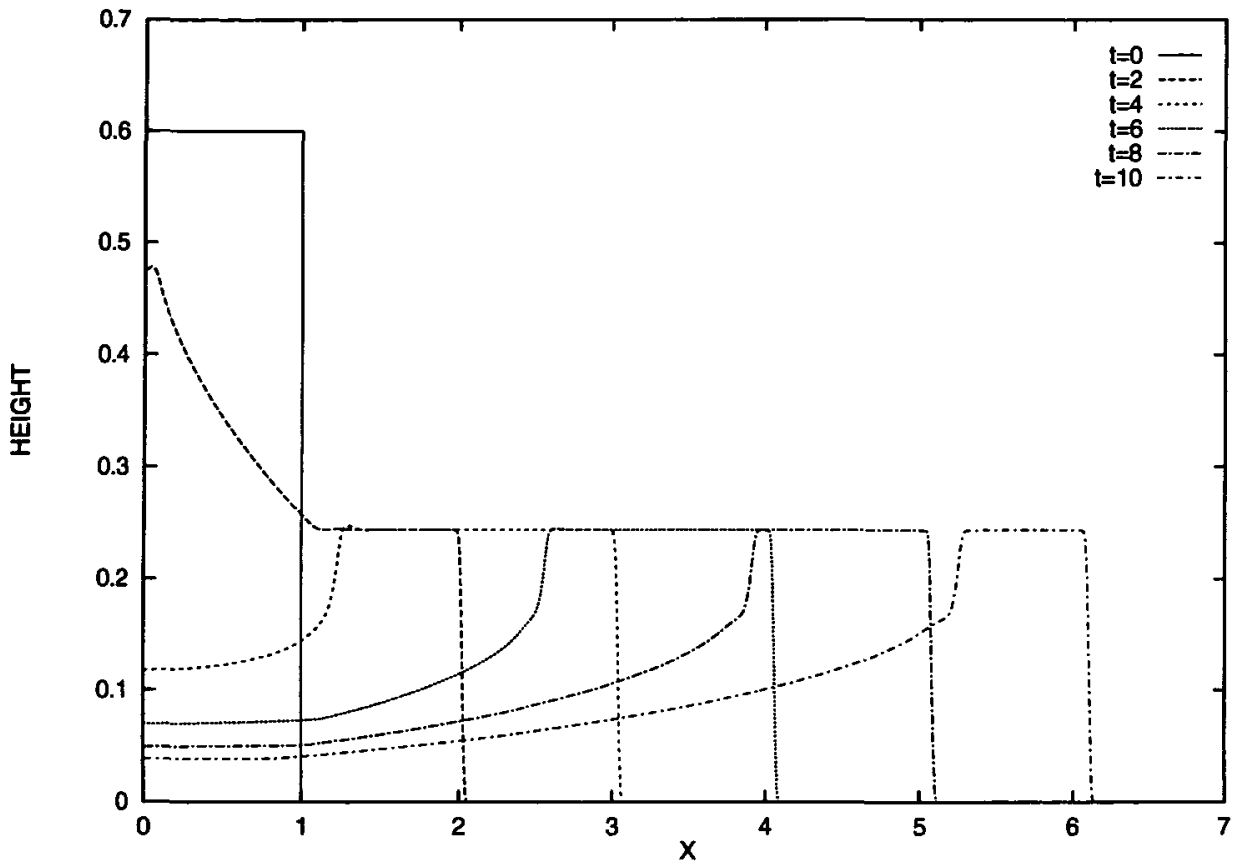

FIGURE 8A. Time evolution of solutions to the weak-stratification equations (2.11) with $h_{0}=0.6$.

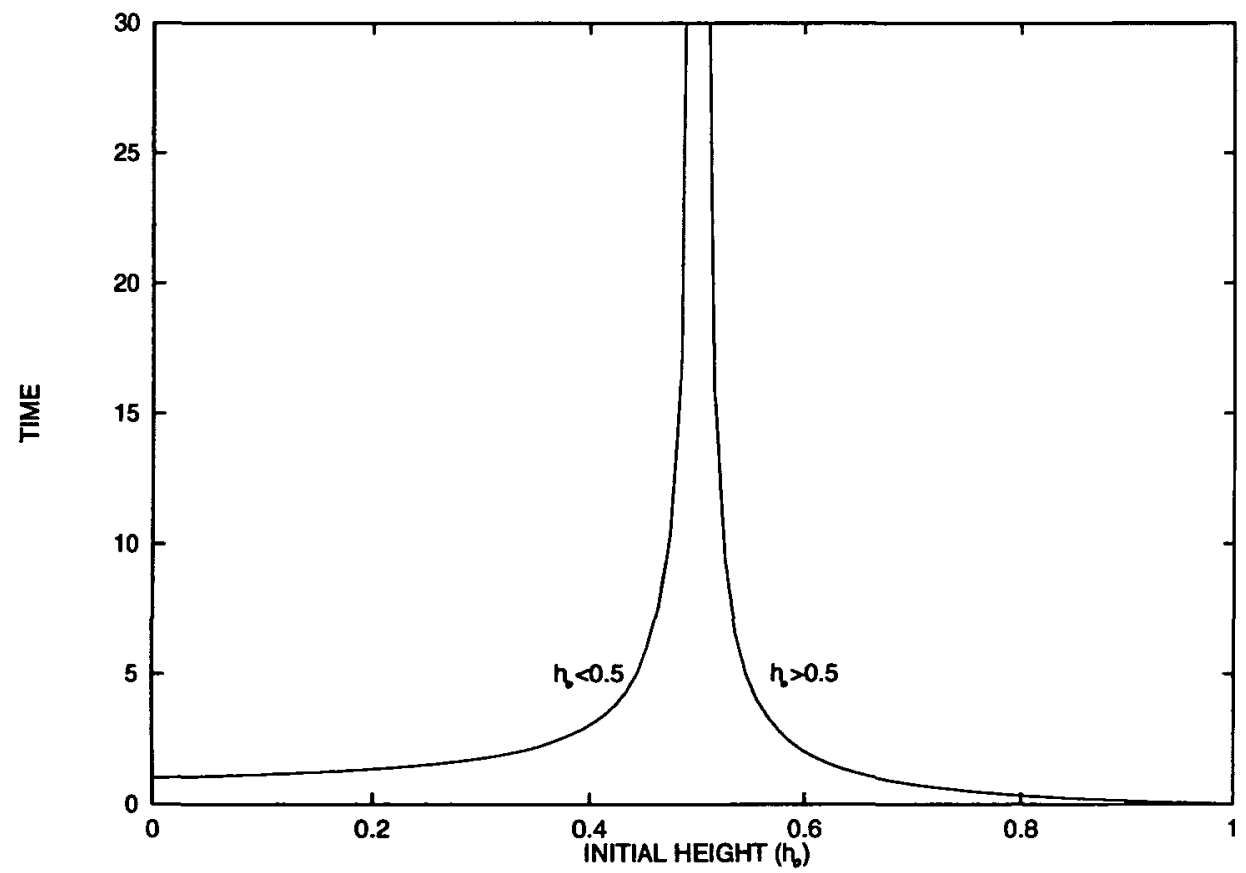

FIGURE 8B. Breaking time as a function of initial height $h_{0}$ for the weak-stratification equations (2.11) with the assumption that $\min _{\xi} \sigma_{0}^{ \pm \prime}(\xi) \simeq 1$. 
Using the initial data decomposition (5.2) and the orthonormality requirement (4.8) we see that for $\hat{\mathbf{u}}(x)=(\hat{u}(x), \hat{h}(x))$ we have

$$
\sigma_{0}^{ \pm^{\prime}}= \pm \sqrt{\frac{h_{0}}{1-h_{0}}} \hat{u}^{\prime}(x)+\hat{h}^{\prime}(x) .
$$

To interpret these results, we consider a right travelling wave corresponding to $\lambda^{+}$at the interface of the layers and determine the breaking time for a typical height profile. From (5.7) and (5.8) we see that for $0<h_{0}<\frac{1}{2}$, a shock will form in positive time only for ${\sigma_{0}^{+\prime}}^{+}<0$. Conversely, for $\frac{1}{2}<h_{0}<1$ a shock will form in positive time only for ${\sigma_{0}^{+\prime}}^{+} 0$. In this latter case, a necessary condition for shock formation with initial data decomposition (5.9) is then

$$
\hat{h}^{\prime}(x)>-\sqrt{\frac{h_{0}}{1-h_{0}}} \hat{u}^{\prime}(x) .
$$

Although not shown in Figure 2, the typical small variations in $\hat{u}$ allow equation (5.10) to be replaced by the simpler restriction $\hat{h}^{\prime}>0$. Summarizing, for $h_{0}>\frac{1}{2}$ we expect to see shock formation in a finite time for an increase in interface height with increasing $x$. This can be seen in Figure 8A. Also, using a value of $\hat{h}^{\prime}=1$, the relationship between breaking time and initial interface height is plotted in Figure 8B.

For the thin lower and upper-layer systems, a similar expansion procedure can be carried out about a nonzero constant initial state. Here, the interaction coefficients do not change sign and are easily found to be $\Gamma^{ \pm}=3 / 4$. Shock formation in this case can be analyzed similarly but is uncommon due to the usually low variation in interface height.

\section{Concluding Remarks}

In this paper we have outlined the derivation of model equations for three distinguished limits in two-layer gravity current analysis. These are the weakly-stratified case, and the cases of thin upper or lower layers. All of these cases were examined numerically using a second-order relaxation scheme for conservation laws. This numerical method is well suited to the hyperbolic calculations completed here, although strict hyperbolicity is not necessary in theory [8]. For the weakly-stratified case, we found an invariant region in phase space in which the equations were strictly hyperbolic, which coincided with the region given for subcritical flow in terms of a Froude number. In the case of perturbations about a constant state, shocks may occur at the interface of the fluids and the breaking times for these shocks have been derived. 


\section{References}

[1] T. B. Benjamin, "Gravity currents and related phenomena", J. Fluid Mech. 31 (1968) 209-248.

[2] R. T. Bonnecaze, H. E. Huppert and J. R. Lister, "Particle-driven gravity currents", J. Fluid Mech. 250 (1993) 339-369.

[3] S. J. D. D'Alessio, T. B. Moodie, J. P. Pascal and G. E. Swaters, "Gravity currents produced by sudden release of a fixed volume of heavy fluid", Stud. Appl. Math. 96 (1996) 359-385.

[4] S. J. D. D'Alessio, T. B. Moodie, J. P. Pascal and G. E. Swaters, "Intrusive gravity currents", Stud. Appl. Math. 98 (1997) 19-46.

[5] Y. He and T. B. Moodie, "The signaling problem in nonlinear hyperbolic wave theory", Stud. Appl. Math. 91 (1991) 215-245.

[6] D. Hoff, "Invariant regions for systems of conservation laws", Trans. Amer. Math. Soc. 289 (1985) 591-610.

[7] D. P. Hoult, "Oil spreading on the sea", Ann. Rev. Fluid Mech. 4 (1972) 341-368.

[8] S. Jin and Z. Xin, "The relaxation schemes for systems of conservation laws in arbitrary space dimensions", Comm. Pure and Appl. Math. 48 (1995) 235-276.

[9] T. von Kármán, "The engineer grapples with nonlinear problems", Bull. Amer. Math. Soc. 46 (1940) 615-683.

[10] G. A. Lawrence, "On the hydraulics of Boussinesq and non-Boussinesq two-layer flows", J. Fluid Mech. 215 (1990) 457-480.

[11] R. J. LeVeque, Numerical Methods for Conservation Laws (Birkhäuser-Verlag, Basel, 1992).

[12] A. Majda and R. Rosales, "Resonantly interacting weakly nonlinear hyperbolic waves, I: A single space variable", Stud. Appl. Math 71 (1984) 149-179.

[13] J. Pedlosky, Geophysical Fluid Dynamics (Springer-Verlag, New York, 1987).

[14] J. E. Simpson, Gravity Currents: In the Environment and the Laboratory (Ellis Horwood, Chichester, 1987).

[15] G. B. Whitham, Linear and Nonlinear Waves (J. Wiley and Sons, New York, 1974). 